\title{
Proactive TCP Mechanism to Improve Handover Performance in Mobile Satellite and Terrestrial Networks
}

\author{
*Preetida Vinayakray-Jani \\ Dhirubhai Ambani Institute of Information and \\ Communication Technology \\ Gandhinagar, India \\ ${ }^{*}$ Corresponding Author
}

\author{
Sugata Sanyal \\ School of Technology and Computer Science, \\ Tata Institute of Fundamental Research, \\ Mumbai, India
}

\begin{abstract}
Emerging standardization of Geo Mobile Radio (GMR-1) for space segment technology like satellite system is having strong resemblance to terrestrial GSM (Global System for Mobile communications) at the upper protocol layers of OSI and TCP (Transmission Control Protocol) is one of them. This space segment technology as well as terrestrial technology, is characterized by periodic variations in communication properties and coverage, causing the termination of ongoing call as connections of Mobile Nodes (MN) alter stochastically. Although provisions are made to provide efficient communication infrastructure this hybrid space and terrestrial networks must ensure the end-to-end network performance so that $\mathrm{MN}$ can move seamlessly among these networks. However from connectivity point of view current TCP performance has not been engineered for mobility events in multi-radio $\mathrm{MN}$, when a sudden change in connectivity, due to handover, occurs. While there are protocols to maintain the connection continuity on mobility events, such as Mobile IP (MIP) and Host Identity Protocol (HIP), TCP performance engineering had less attention. TCP is implemented as a separate component in an operating system, and is therefore often unaware of the mobility events or the nature of multiradios' communication. This paper aims to improve TCP communication performance in Mobile satellite and terrestrial networks.
\end{abstract}

\section{General Terms}

Satellite-Terrestrial Networks, Transmission Control Protocol, Inter-system Handover

\section{Keywords}

Satellite-Terrestrial Networks, TCP, Inter-System Handover.

\section{INTRODUCTION}

Traditional terrestrial wireless or cellular networks provide mobile communication services but they remain limited to geographic coverage. High-speed vehicular mobile nodes (e.g. passengers with mobile phone travelling via high-speed train or car etc.) encounter the problem of network connectivity in rural or remote area where terrestrial cellular or wireless network connectivity is either limited or not feasible. In fact, there are areas like tunnels, closed mines, small obstacles, where satellite connectivity suffers from non-line-of-sight events or fading.

Thus, high-speed mobile nodes impose severe constraints on terrestrial or satellite system in terms of Doppler Effect, handover frequency and handover execution time.
Future satellite systems like GMR (Geo Mobile Radio)/GMPRS (Geo Mobile Packet Radio Service) is an optimized version of GSM (Global System for Mobile communication) protocol designed for mobile communication. Geo Stationary Mobile system like Geostationary Orbit Earth (GEO) satellites, will work as an important component in data communication network. These satellites are using multiple spot antennas, where satellite footprint covered on earth partitioned in small cells called spotbeams. Transfer of ongoing connection to a new spotbeam or satellite considered as a link layer handover.

Similarly, as a counterpart terrestrial systems like GSM/GPRS (General Packet Radio Service) optimized to 3G for mobile communication. These satellite systems intend to complement and extend the existing terrestrial network to provide complete coverage while incorporated with Internet technology for data communication $[1,2]$.

Future global communication scenarios must offer fast and integrated services to ubiquitous users, anytime, anywhere, hence the seamless handover from terrestrial network to satellite or vice versa becomes essential.

In this focused heterogeneous networking environment, the Mobile Node (MN) often encounters the instant changes in link performance and coverage [3]. However such abrupt changes in link quality or coverage either interrupts or terminates the ongoing TCP connection and eventually deteriorates the performance of the ongoing application flows, including delaying the registration process for mobility protocols Meanwhile, there is no classical TCP stack, which can simultaneously handle connections efficiently on these paths with different characteristics. Applications have different networks and TCP requirements, such as Telnet protocol values short response time (delay sensitivity), but ftp protocol values high throughput (delay tolerant). Moreover, application run on phone or small PDA has limited power hence TCP should try best to avoid unnecessary retransmission to save energy.

Therefore, a proactive TCP mechanism, aware of the flows' characteristics and ongoing stochastic changes in radio networks is an effective and efficient approach to solve such problem [4]. Hence, here the attempt is to make the seamless handover more efficient by making TCP transmission windows to adapt the network environment proactively that can prevent congestion and buffer overflow situations effectively, making Mobile Nodes (MN) to experience the seamless mobility with consistent quality. 
However, there are several approaches to deal with disruption in connectivity issue like Delay/disruption tolerant Networking [5], Proxy enhancing Proxy (PEP) [6], Accelerated TCP [7], or combination of these approaches.

The proposed proactive handover mechanism in this paper enables the $\mathrm{MN}$ to perform inter-system handover to maintain the seamless connectivity, where connection to target network is made before breaking the current one.

The paper organized as follows; the section 2 provides literature review in background. Section 3 shows reference architecture of network connectivity where mobile node handovers from GPRS to WLAN and then to satellite is shown. The TCP improvement mechanism has been described through timeline schematic view in subsections 3.1 and 3.2 showing MN handovers from terrestrial networks (GPRS/WLAN) to Satellite and vice versa. Finally concluding remarks with future work is described in section 4 followed by references in section 5

\section{BACKGROUND}

There has been past work to adjust TCP's receive window to suppress the TCP sender from sending further data, and to adjust the receive window to be better suitable for slow modem link. However, this work has targeted environments with static and fixed setup, and has not been applied in a dynamic network environment where different types of applications and multiple available networks with terminal mobility have been shown in $[8,9]$. This approach targeted for mobile environment where TCP window size dynamically evaluated

Another proposed approach where a handover prioritization scheme for different channel allocation techniques in satellite network defined, where handover requests are queued for predefined time interval [10]. If there are no resources available during this interval then ongoing connectivity of services will terminated. Also [11] proposes time based channel reservation by reserving channel in next targeting spotbeam to improve handover performance.

There has been few research approaches on adjusting the TCP's slow-start threshold based on explicit indication of available bandwidth on the path. Focused on TCP receiver that is in most cases the mobile terminal, this requires modifications to the TCP sender side that is often a fixed server and therefore inaccessible to the mobile terminal vendors [12]. Therefore, the earlier work does not overlap with the solution this paper suggests.

There is a paper to set the receiver window to zero during the handoff to suspend the sender and avoid packet losses [13]. Here the approach was to set the receiver window directly based on bandwidth-delay product. There is some past work on receive buffer auto tuning based on bandwidth-delay product, but this does not discuss the vertical handoff [14]. This proposed work requires TCP modification on receiver side where as the sender side is required to enter in slow start phase following handover. In [15] the proposed work suggests that buffer of all links configured with maximum bandwidthdelay product. However, such work requires reliance on operator and this is somewhat difficult to justify when there is a link with low bandwidth-delay product, this may cause excess queuing.

The inter segment handover proposed in [16] considers the different phases of handover like initiation, decision and execution for different handover controlling schemes, based on where the handover initiation or decision phases are executed, in Mobile node or in network.

The work proposed in [17] closes the congestion window (cwnd) at the sender before the inter-system handover execution takes place. When inter-system handover reestablishes the connection in new TCP segment, cwnd is reset to 1 and Slow-Start Threshold (ssthresh) is set equal to estimated bandwidth for the new link. However, this method does not seem to provide seamless handover from Wi-Fi to satellite.

\section{REFERENCE ARCHITECTURE}

Reference architecture is shown in Figure 1 where multi-mode Mobile Node (MN) encounters the connectivity signals from three different networks when on move. While being on vehicle it receives connectivity from $3 \mathrm{G}$ terrestrial network in urban area. Once vehicle approaches a tunnel, $\mathrm{MN}$ switches to $\mathrm{Wi}-\mathrm{Fi}$ due to inter-system handover from $3 \mathrm{G}$ to $\mathrm{Wi}-\mathrm{Fi}$. Once the vehicle exits from tunnel and enters in rural area, $\mathrm{MN}$ gets connected through geostationary satellite. MN receives the IP address from corresponding network while being connected.

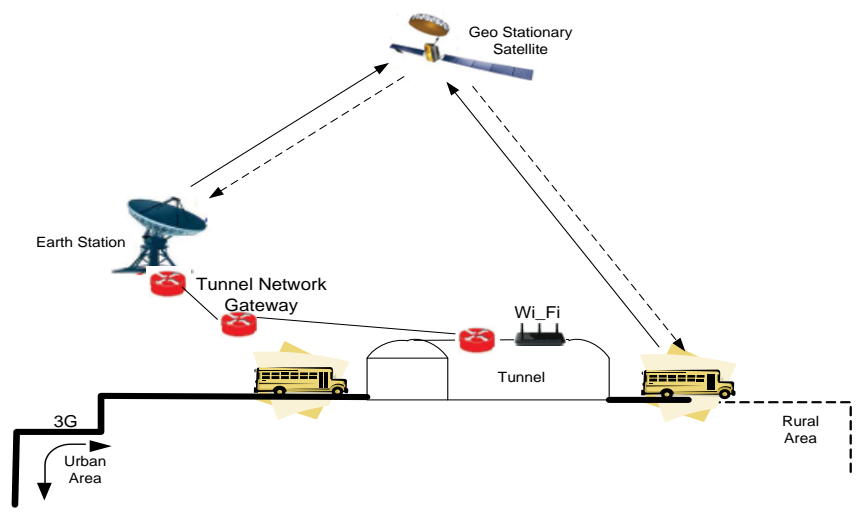

Figure 1 Reference Architecture: Network Connectivity

From network connectivity point of view the mobility and inter-system handover, events managed at both Layer 2 (L2) and Layer 3 (L3) for the selection of new network link and for configuring system with new IP address. Limiting the scope of solution to L3 perspective work adopts the Mobile Internet Protocol Version 6 (MIPv6). It accompanies an efficient rerouting feature. However, the proposed solution is also applicable to MIPv4. These adopted mobility protocol requires the registration of received IP addresses in corresponding Home Agent (HA), so MN can maintain end to end connectivity while on the move.

Alternatively, Proxy Mobile IP (P-MIP) is located within the immediate gateway/router of terrestrial cellular networks' Service Access Point (SAP) in base station or Wi-Fi network's SAP in access router/gateway or satellite earth station interface with terrestrial GMR1. Interface with SAP required to reroute the Binding Update (BU) towards HA for corresponding $\mathrm{MN}$. Thus if $\mathrm{MN}$ is not optimized with MIPv6/MIPv4, it is able to maintain end-to-end connectivity, through P-MIP.

However, periodic variation in radio quality and high mobility of MN, significantly affects the TCP performance. This also makes connection of $\mathrm{MN}$ to any network to alter stochastically. 
Few parameters that considered during handover phase as they play important role for TCP performance. Such parameters include duration of handover phase, the coverage area overlapped with two different networks, speed of vehicle and buffer size available at sender, receiver and network. Aim of this paper is to provide TCP performance improvement in two possible inter-system handover cases in mobile node, from terrestrial network to satellite and from satellite to terrestrial network.

Note that proposed following approach is similar to [18], but remained limited to terrestrial network only. Our approach extended to satellite network communication as well.

\subsection{GPRS/WLAN->Satellite}

When $\mathrm{MN}$ detects the imminent possibility for handover for currently active flow(s) it can disseminate the window size to peer or Corresponding Node $(\mathrm{CN})$ and that can be set as:

$$
\mathrm{W}_{\text {Default }}>\mathrm{SATWIN}_{\max } \geq \mathrm{W}_{\mathrm{REC}}
$$

When previously connected to the Satellite network, the MN can try to estimate the maximum window size offered by satellite through bandwidth-delay product of the wireless link. $\mathrm{MN}$ also has the default value $\left(\mathrm{W}_{\text {Default }}\right)$ of the maximum window size that is usually larger than the bandwidth-delay product of the path. Hence it is quite obvious that in a network such as Satellite or GPRS the maximum bandwidth delay product offered is considered to be less than the default value used by TCP. Hence MN advertises a smaller window ( $\left.\mathrm{W}_{\mathrm{REC}}\right)$

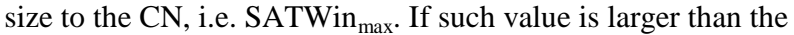
default value, the TCP sender can flood the cellular network that can cause excess queuing at $2 \mathrm{G} / 3 \mathrm{G}$ base station rather than buffer overflow.

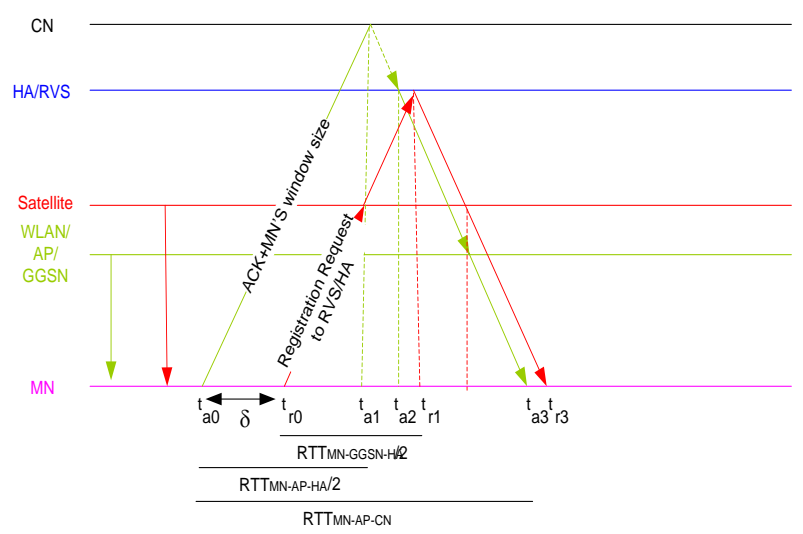

Figure 2 Operational View of Inter-system Handover

Alternatively one can assume that $\mathrm{MN}$ has some previously estimated value of Satellite bandwidth delay product. Using this cached information $\mathrm{MN}$ selects the value for the receive window size and advertises it to the $\mathrm{CN}$ in a TCP acknowledgement (ACK) packet at $\mathrm{t}_{\mathrm{a} 0}$, as shown in figure 2 .

Hence MN will deliver new window size before initiating its registration of a new location address to the RVS or HA. Upon receiving the window advertisement for future flows at $\mathrm{t}_{\mathrm{a} 1} \mathrm{CN}$ limits its data transmission rate as per the new received window size. Thus new TCP window adjustment at the $\mathrm{CN}$ actively reduces the network congestion by controlling the rate at which the $\mathrm{CN}$ communicates. Subsequently MN delays its registration process of received new location for a period of $\delta$ and sends the registration request at $\mathrm{t}_{\mathrm{r} 0}$.
After registration at RVS/HA at $t_{r}$, RVS/HA redirects all the packets to new located address. The last TCP packet sent just before the use of the new window size encountered by the $\mathrm{CN}$ is received by RVS/HA at time $t_{\mathrm{a} 2}$ and forwarded to the WLAN link. Therefore if $t_{\mathrm{r} 1} \ll \mathrm{t}_{\mathrm{a} 2}$ then some packets are redirected to the GPRS network, before the influence of new window takes place. This will create buffer queue to be filled to overflowing. To avoid this problem, the value of $\delta$ should be chosen so that $t_{r 1} \gg t_{a 2}$. Hence $M N$ should determine the value of $\delta$. It can be seen from the figure 2 ,

$\delta \leq \mathrm{RTT}_{\text {MN-SAT-CN }}-0.5\left(\mathrm{RTT}_{\mathrm{MN}-\mathrm{SAT}-\mathrm{RVS} / \mathrm{HA}}+\mathrm{RTT}_{\mathrm{MN}-\mathrm{GGSN}-/ \mathrm{HA}}\right)$

Once the possibility of handover is detected, then $\delta$ interval handover decision can be made and inter-system handover execution phase starts. As a result then either MN or P-MIP interface in GMR1 interface of satellite generates registration request through $\mathrm{BU}$ towards $\mathrm{HA}$. The $\mathrm{MN}$ or P-MIP will receive $\mathrm{BU}$ ack at $\mathrm{t}_{\mathrm{r} 3}$. It is important to note that $\mathrm{HA}$ is capable to register more than one binding update requests for given $\mathrm{MN}$. Thus if $\mathrm{MN}$ remains connected to geostationary satellite network then seamless beam handover or intrasystem handover requires only L2 handover as IP address received by $\mathrm{MN}$ from satellite network remains same.

Thus similar type of analogy can be applied to intra-system handover also.

\subsection{Satellite->GPRS/WLAN}

When handover from Satellite to GPRS/WLAN or any terrestrial network occurs, new packets reach the TCP receiver through the GPRS/WLAN link while some of the older packets are still in transit over the satellite network. Because packets arrive at the receiver in wrong order, the receiver generates duplicate acknowledgements that cause a retransmission at the TCP sender. This retransmission is unnecessary, because no packets have been lost and it simply degrades TCP throughput. Therefore, prior to handover from satellite to terrestrial network, it would be useful to temporarily close the TCP's advertised window for a short while until all outstanding packets from satellite link have arrived, to prevent packet reordering from happening.

However reordering approach defined in [19] is a sender and receiver side modification that allows managing the arrival of out-of-sequence packets due to the intersystem handover from high RTT (Round Trip Time) satellite network to low RTT WLAN (WLAN). Instead of DupACKS (Duplicate Acknowledgements), ACKs are sent and a congestion avoidance phase is performed during the interval for which out-of-sequence packets are received

The handover from satellite to GPRS/WLAN encounters the problem of under-utilization; as packet processing in satellite may take longer time due to the absence of sufficient duplicate ACKs to trigger the fast transmission of the packets over the faster GPRS/WLAN. Hence even though the advertised window size is larger, the packet remains enqueued for a long time in satellite network due to lack of duplicate ACK packets from GPRS/WLAN. Smaller bandwidth (compared to satellite) of GPRS/WLAN capacity can be underutilized because the satellite link may use significantly smaller size of advertised window than what the bandwidthdelay product of GPRS/WLAN would require. However there are attempts made by NASA to make use of TCP with Large Window (LW) - TCP-LW 
To handle the packet loss while performing the handover to GPRS/WLAN, MN increases the current satellite window size with bandwidth-delay product. The window should be set appropriately to avoid fragmentation. This will permit $\mathrm{CN}$ to send more data benefiting from the fact that a $\mathrm{CN}$ can continue to increase its congestion window size when the amount maximum threshold of received window size is crossed. This may create the burst of data packets as well as duplicate ACKs that can trigger the fast transmission of the packets queued at Satellite. Increasing the advertised window size more than two segments may make $\mathrm{CN}$ to overflow the network. Meanwhile the gradual increase in advertised receiver window size makes consistent utilization of WLAN network, once handover performed.

\section{CONCLUSION}

The TCP window advertisement permits end-systems to actively reduce the congestion (in any TCP queue) by controlling the rate at which end-systems communicate. While performing handover from fast network to slow network, the network heads toward the congestion, the TCP flows are rate shaped by adjusting the receivers' advertised window size and simultaneously slowing down the return of the ACKs, as endsystems communications align toward the consistent possible rate. Meanwhile, when MN is supported with multi-homing mobility feature, it is very likely that such advanced mobility protocols are able to move simultaneously running traffic flows, independently from one access interface to another. Hence simultaneous flows, relevant to different or single applications may have different throughput or bandwidth requirements. Such requirements are directly influenced by the received window size parameter TcpWindowSize. Therefore proposed proactive handover engine allocates the proportional TCP window size by considering the type of the access network availability, single flow requirement and available buffer capacity. In proposed innovative work the MN advertises Maximum TcpWindowSize to $\mathrm{CN}$ along the ACK at appropriate time before initiating for handover and it will follow such analogy for subsequent TCP packets also. Hence proposed method is applicable when both end-systems are mobile. The proposed method is versatile as it can operate over any link layer technology e.g. Wi-MAX, WCDMA, Satellite etc. and hence it supports the Media Independent handover standardization activity, as well. The proposed method does not require any modification or enhancement in access networks.

However, further study is needed to compare the proposed work with the NASA Space Communications Protocol Specification-Transport protocol (SCPS-TP), including transport layer alternative algorithm proposed in [20]. As satellite is an open space global communication, TCP has to maintain its reliability property even when network is prone to packet loss, rendering and duplication. Besides, the TCP performance improvement also requires the $\mathrm{MN}$ to measure the round trip time more accurately. Hence it is desirable to implement reliability with an optimum flow control; this gives scope for Steganographic Coding [21]. As a part of further enhancement of proposed solution, various TCP header fields will be examined so that they can be further strengthened with application of Steganography, to maintain the confidentiality of TCP signalling and data.

\section{REFERENCES}

[1] Bhasin K. and Hayden J. L., "Space Internet Architectures and Technologies for NASA enterprises", International Journal of Satellite Communications, Special Issue: Communications for Space Missions, Volume 20, Issue 5, pages 311-332, September/October 2002

[2] Leung K., Shell D., Ivancic W., Stewart D.H., Bell T. L., and Kachmar B., "Application of mobile-IP to Space and Aeronautical Networks," IEEE Proceedings of Aerospace Conference, Big Sky, MT,USA, Vol. 2, pp,1027-1033, 10-17 March, 2001, doi: 10.1109/AERO.2001.931283

[3] Falk A., Jasapara N., "Can a Satellite be an Internet router?" Available at: http://scholar.google.com/scholar? $\mathrm{q}=\mathrm{Can}+\mathrm{a}+$ Satellite+be+an+Internet+router \&btnG=\&hl=e n\&as_sdt $=0 \% 2 \mathrm{C} 5$

[4] Luglio M., Roseti C., Savone G., Zampognaro F., "Cross-Layer Architecture for a Satellite-Wi-Fi Efficient Handover" IEEE Transactions on Vehicular Technology, Vol. 58, No.6 pp.2990-3001, July, 2009, doi: 10.1109/TVT.2008.2011274.

[5] Wang R., Wu X., Zhang Q., Taleb T., Zhang Z. and Hou J., "Experimental Evaluation of TCP based DTN for Cislunar Communication in Presence of Long Link Disruption" Special Issue on opportunistic and delay tolerant networks of EURASIP Journal on Wireless Communications and Networking, Vol. 2011, January, 2011, pp. 1-11, Article No. 8. Doi: $10.1155 / 2011 / 720671$.

[6] Dubois E., Fasson J., Donny C. and Chaput E., "Enhancing TCP based Communication in Mobile satellite Scenarios: TCP PEPs Issues and Solutions", $5^{\text {th }}$ Advanced Satellite Multimedia Systems Conference and the $11^{\text {th }}$ Signal Processing for Space Communications Workshop (ASMA/SPSC 2010), Sept. 13-15, 2010. Cagliari, Italy

[7] Giambene G., Marchi S., and Kota S., "TCP Performance Issues in Satellite and Wi-Fi Hybrid Networks for High-Speed Trains", ICST Transactions on Ubiquitous Environnements, Jan -Mar, 2012, Vol. 12, Issue 1-3, pp. 1-12.

[8] Kim H., Lee S., "Improving TCP Performance for Vertical Handover in Heterogeneous Wireless Networks", International Journal of Communication Systems, Vol. 22, Issue 8, pp.1001-1021, August, 2009

[9] Li Y.T., Leith D., Shorten R. N., "Experimental Evaluation of TCP Protocols for High-Speed Networks", IEEE/ACM Transactions on Networking, Vol. 15, No. 5, pp.1109-1122, October, 2007.

[10] Del Re E., Fantacci R. and Giambene G., "Efficient Dynamic Channel Allocation Techniques with Handover Queuing for mobile Satellite Networks", IEEE Journal on selected Areas in Communications, Vol.13, No 2, pp.397-405, February, 1995.

[11] Boukhatem L., Gaiti D. and Pujolle G., "A time- based reservation scheme for managing handovers in satellite system", International Journal of Network Management, Vol. 13, No 2, pp. 139-145, March/April 2003, doi $>10.1002 /$ nem.467. 
[12] Allman M., Glover D. and Sanchez L., "Enhancing TCP over Satellite Channel using Standard Mechanisms", RFC 2488 (Best Current practice), January, 1999. Available at: http://tools.ietf.org/html/rfc2488

[13] Vidales P., Patanapongpibul L., Mapp G. and Hopper A., "Experiences with Heterogeneous Wireless Networks, Unveiling the Challenges" Available at: http://www.cl.cam.ac.uk/research/dtg/publications/public /pav25/HetNets04-Vidales.pdf

[14] "TCP auto-tuning zoo" Available at: http://www.csm.ornl.gov/ dunigan/net100/auto.html

[15] Gurtov A., Passoja M., Aalto O. and Raitola M., "MultiLayer Protocol Tracing in a GPRS Network" Available at: http://www.cs.helsinki.fi/u/gurtov/papers/vtc02.pdf

[16] Leo M. and Luglio M., "Intersegment Handover between Terrestrial and Satellite Segments: Analysis and Performance Evaluation Through Simulation," IEEE Transactions on Vehicular Technology, Vol. 50, No. 3, pp. 750-766, May, 2001, doi: 10.1109/25.933310.

[17] Luglio M., Roseti C., Savone G., and Zampognaro F., "Cross-layer Architecture for a Satellite-Wi-Fi efficient
Handover", IEEE Transaction on Vehicular Technology, Vol. 58, No.6, July, 2009, pp. 2990-3001,

[18] (57488-US-NP) Status: Patent - PAT, Title: A MOBILE NODE, A METHOD OR HANDOVER AND A COMPUTER PROGRAM (IPR Title: End Device Controlled Proactive TCP mechanism for Inter-system Handover). Inventor: Preetida Vinayakray-Jani, Pasi Sarolahti.

[19] Kim H., Lee S., "Improving TCP Performance for Vertical Handover in Heterogeneous Wireless Network", International Journal of Communication Systems, Vol. 22, Issue 8, August, 2009, pp. 1001-1021.

[20] Wang R, Gutha B.and Rapet P., "Window-based and Rate-based Transmission Control Protocol Mechanisms over Space-Internet Links", IEEE Transaction on Aerospace and Electronics Systems, Vol. 44, No 5, 2007, pp. 1109-1122.

[21] Das S., Das S., Bandyopadhyay B. and Sanyal S., "Steganography and Steganalysis: Different Approaches", International Journal of Computers, Information Technology and Engineering (IJCITAE), Vol. 2, No 1, June 2008, Serial Publications. 